\title{
Reaching Beyond Arm Length in Normal Aging: Adaptation of Hand Trajectory and Dynamic Equilibrium
}

\author{
Christos Paizis, Charalambos Papaxanthis, \\ Bastien Berret \\ Université de Bourgogne
}

\author{
Thierry Pozzo \\ Université de Bourgogne and Italian Institute of Technology
}

\begin{abstract}
The authors investigated the influence of normal aging upon equilibrium and kinematics features during a whole-body task. Eight young ( $23 \pm 1.51$ years) and eight elderly ( $74.5 \pm 4.5$ years) adults reached from a standing position an object placed in front of them on the ground. The authors found smaller Center of Masse (CoM) and Center of Pressure (CoP) antero-posterior displacements in elderly than in young adults. Wrist paths were curved in young but straight in elderly adults. Wrist peak velocity and duration were respectively lower and greater in elderly compared to young adults. However, PrincipalComponent-Analysis did not reveal differences in angle coordination between the two groups, suggesting so that modifications in equilibrium and wrist kinematics reflect an adaptation process that compensates age-related physiological changes. The authors hypothesized that equilibrium preservation in elderly contributes to wrist kinematics modifications. The authors verified this premise by placing young adults under equilibrium restrictions (reduced base of support) and observing that they reproduced the behavior of elderly adults. The authors propose that wrist kinematics is equilibrium dependent and that such a strategy is included in the motor plan of elderly adults.
\end{abstract}

Keywords: aging, motor coordination, equilibrium, wrist kinematics, center of mass
It is now well established that efficient and precise control of equilibrium and movement decrease with aging and often cause serious balance disturbances (Alexander, 1994; Morasso, Baratto, Capra, \& Spada, 1999; Mourey, Pozzo, Rouhier-Marcer, \& Didier, 1998; Salthouse \& Somberg, 1982; Vaillant et al., 2008). Deterioration of equilibrium function is considered by geriatrics as the primary cause of fall in elderly. At the peripheral level, loss of muscle strength, slowing of contraction speed and decline in several aspects of proprioceptive sensitivity, which are wellknown changes with age (Narici, Maganaris, Reeves, \& Capodaglio, 2003; Simoneau, Billot, Martin, \& Van Hoecke, 2007; Verschueren, Brumagne, Swinnen, \& Cordo, 2002; Whipple, Wolfson, \& Amerman, 1987), may alter the capacity to plan a movement and to produce rapid changes in force for balance corrections in elderly. At the central-planning level, studies have shown that elderly seemed to prefer balance strategies different from those used by young adults. In particular, they used the "ankle" strategy less than young adults (Amiridis, Hatzitaki, \& Arabatzi, 2003). Less postural control and extreme CoM displacement were also observed in elderly during static equilibrium or unexpected perturbation of the standing posture. Fur-

Christos Paizis, Charalambos Papaxanthis, and Bastien Berret, Université de Bourgogne, Campus Universitaire, Dijon, France; Thierry Pozzo, Université de Bourgogne, Campus Universitaire, Dijon, France, and Italian Institute of Technology, Genova, Italy.

This work was supported by the Conseil Regional de Bourgogne and by the INSERM.

Correspondence concerning this article should be addressed to Prof. Charalambos Papaxanthis, INSERM/U877, Université de Bourgogne, Campus Universitaire, BP 27877, Dijon 21078, France. E-mail: charalambos. papaxanthis@u-bourgogne.fr thermore, elderly adults show coactivation of antagonist muscles (Klein, Rice, \& Marsh, 2001; Seidler, Alberts, \& Stelmach, 2002) lengthened deceleration curves, lower peak velocities and secondary corrective submovements during movements involving the arm (Bellgrove, Phillips, Bradshaw, \& Gallucci, 1998; Ketcham, Seidler, Van Gemmert, \& Stelmach, 2002; Seidler-Dobrin \& Stelmach, 1998).

Until now, equilibrium function and control of arm movements in elderly adults have been studied separately although; CoM and upper arm displacements are mechanically linked during whole body movements. This dissociation of postural and focal aspects of movement has several limitations because it precludes the understanding of their concomitant influence in movement control in the aging motor system. Evaluation of equilibrium using unexpected perturbation mainly tests reflex compensation, while it is now widely recognized that equilibrium is not the sum of several righting reflexes (Horak \& McPherson, 1996) but integrates the interaction of multiple sensorimotor processes. Additionally, the patterns of $\mathrm{CoM}$ displacement recorded during standing posture are very small and frequently failed to show instability in elderly. On the other hand, investigating upper arm movements in elderly adults may be useful for understating the decline of the control and the planning processes with age. However, it does not indicate how similar arm movements are controlled and executed under equilibrium constraints. To what extent does equilibrium influences arm movements in elderly and vice versa?

The main purpose of this study is to better understand equilibrium function and movement coordination in elderly by means of a whole body goal oriented task. Paradoxically, while aging adults usually do not fall from a quite standing posture but during dynamic condition (Maki et al., 2008; Medell \& Alexander, 2000), the decline with age of high-level neural processes related to dynamic equilibrium has not 
yet received high scientific attention. Before a voluntary movement, anticipatory postural adjustments (APAs) helps to maintain stability by compensating for destabilization associated with the focal motion (Belen'kii, Gurfinkel, \& Pal'tsev, 1967; Lee, 1980). For instance when reaching an object located on the ground the forward CoM displacement because of the arm motion toward the target must be compensated. Moreover, because of the different arm, trunk, and lower limbs inertias and of consecutive complicated joint interactions, a sophisticated control of joint torques is required to prevent postural unbalance. Finally, during such a task the central nervous system (CNS) has to solve two different problems. A hand trajectory toward the extracorporeal target has to be defined among a plethora, whereas at the same time the CoM of the entire body must remain inside the supporting foot area. Therefore, the CNS must finely coordinate the movement of upper and lower body parts. Thus, joint and muscle redundancy and the difficulty of maintaining the CoM inside the supporting base contribute to the complexity of the task. Because of potential effect of aging on subject' capacity to coordinates APAs (Maki, 1993; Woollacott \& Manchester, 1993) one can predict body instability during selfinitiated action in elderly.

Accordingly, a whole body reaching task seems to be a sensitive tool because of the critical conditions imposed by the reach beyond arm length, hence allowing a better understanding of the effect of aging on equilibrium function. The following questions were asked: To what extent the is CoM displaced during a task involving the whole body? Is joint coordination recorded in young subjects preserved in aging adults? Do elderly participants maintain the strong coupling between focal and postural components of the task observed in young adults? The kinematic of the body movement and wrist reaching trajectory, joint angle covariation, and $\mathrm{CoP}$ and $\mathrm{CoM}$ trajectories were analyzed to answer these questions.

\section{Materials and Method}

\section{Participants}

Eight young and eight elderly adults without any neurological and musculoskeletal diseases or impairments voluntarily participated in the current study. Written consents were obtained from all the participants and a local ethics committee approved the experimental protocol that was carried out in agreement with legal requirements and international norms (Declaration of Helsinki, 1964). The young adults (four females and four males; mean age: $23 \pm 1.51$ years; mean height: $170 \pm 8 \mathrm{~cm}$; mean weight: $62.8 \pm$ 7.4) were students from the University of Burgundy. The elderly adults (four females and four males; mean age: $74.5 \pm 4.5$ years; mean height: $165 \pm 7.5 \mathrm{~cm}$; mean weight: $58.3 \pm 6.5)$ were all retired, had a regular physical activity $(\sim 1.5$ hours 2 days per week, approved by a medical doctor) and at least a daily cognitive activity (reading newspapers, crosswords, or literature). Elderly participants had cognitive evaluation by means of the mini mental state examination test (individual scores $>28$ ).

\section{Motor Task}

Young and elderly participants were initially asked to adopt an erect standing posture, their arms were placed in front of the body (hands crossed, the left palm covering the right hand) both comfortably placed against the body at the level of the navel. From that initial position, participants were requested to make a whole-body movement in the sagittal plane to grasp a wooden dowel $(0.40 \mathrm{~m}$ long, $0.07 \mathrm{~m}$ in diameter, mounted on two semicircular supports of $0.15 \mathrm{~m}$ ) placed at ground level in front of them (5\% of their body height, measured from the distal end of their great toe). No formal indications were given as to the strategy required to make the movement and grasp the object; participants were asked, however, not to support their body weight on the dowel. Participants had to perform the movement at a natural speed ( $\mathrm{N}$ condition) or as fast as possible ( $\mathrm{F}$ condition). Participants accomplished six trials in each condition of speed in a random order. To prevent muscular fatigue accumulation, especially in elderly participants, a rest period of 1 minute was allowed to participants after each trial. Before the experiment, participants had a practice period of 5 minutes in which they performed the motor task four times (two in each speed condition).

\section{Experimental Apparatus}

The experiment took place in a big room $(10 \times 10 \mathrm{~m})$, which was sound-attenuated, temperature regulated $\left(22 \pm 2{ }^{\circ} \mathrm{C}\right)$ and illuminated with homogeneous white light. Whole-body movement analysis was performed by means of an optoelectronic measuring device SMART (BTS, Milan) at a sampling frequency of $120 \mathrm{~Hz}$. Five infra-red-emitting cameras were attached to five vertical poles and were placed circularly on the right side of the participants at a distance of $\sim 4 \mathrm{~m}$. We recorded the displacements of 11 retro-reflective markers $(15 \mathrm{~mm}$ in diameter $)$ placed at the following anatomical sites (see Figure 1): the head (external canthus of the eye and the auditory meatus of the ear), the trunk (the lumbosacral L5-S1 vertebra), the lower limb (the greater trochanter, the knee interstitial joint space, the ankle external malleolus, and the foot fifth metatarsophalangeal), and the upper limb (acromial process of the shoulder, the lateral condyle of the elbow, the styloid process of the wrist and the fifth metacarpophalangeal). Simultaneously with body motion acquisition, anterior-posterior (FX), vertical (FZ), and mediolateral (FY) ground reaction forces as well as the position of the center of foot pressure (CoP) were acquired using an AMTI (Advanced Mechanical Technology Inc. Watertown, MA) force platform (sampling frequency $500 \mathrm{~Hz}$ ).

\section{Data and Statistical Analysis}

Whole body motion normally took place in the sagittal plane. To evaluate the planar assumption we used a Principal Component Analysis (PCA) on the whole 3D data set of each participant (grouping all markers as individual observations). The variance accounted for by the two first principal components (PC) was more than $97 \%$ for all participants, finding that indicates that the motion was in fact performed in a plane. In addition, this plane was almost the sagittal plane defined by the reference frame of the acquisition system (angle between normal vectors of the planes was less than $\left.5^{\circ} \pm 1.3^{\circ}\right)$.

The motion of the 11 markers in the $\mathrm{X}, \mathrm{Y}$, and $\mathrm{Z}$ axes was low-pass filtered (digital second-order Butterworth filter, $6 \mathrm{~Hz}$ cut-off frequency). From the filtered data, we first calculated the 3D motion (position and velocity) of each marker and then the motion of the 

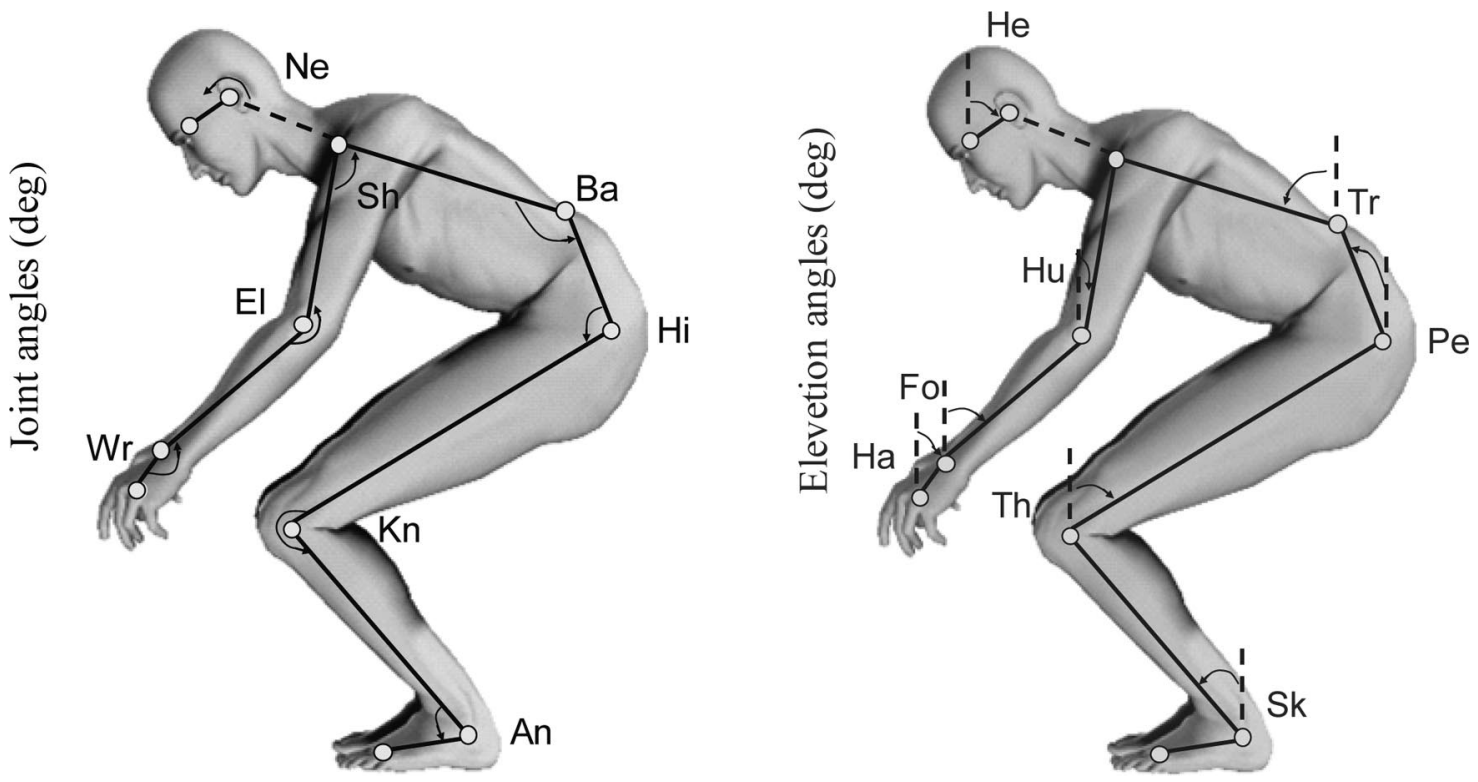

Figure 1. Anatomical position of the markers and angles calculation. Joint angles: ankle (An), knee (Kn), hip $(\mathrm{Hi})$, back $(\mathrm{Ba})$, shoulder $(\mathrm{Sh})$, neck $(\mathrm{Ne})$, elbow $(\mathrm{El})$, and wrist (Wr). Elevations angles: shank (Sk), thigh (Th), pelvis $(\mathrm{Pe})$, trunk $(\mathrm{Tr})$, head $(\mathrm{He})$, humerus $(\mathrm{Hu})$, forearm $(\mathrm{Fo})$, and hand $(\mathrm{Ha})$.

elevation and anatomical angles of the segments involved in the movement. Movement onset and movement end was defined as the time at which linear or angular velocity exceeded and was inferior to $5 \%$ of its peak value. In the present study, we focused our linear analysis on the marker of the wrist, which illustrated nicely the motion of the hand (focal component of the whole body motion). For this marker, we computed the following kinematic parameters: (1) movement duration (MD), (2) peak velocity (V peak), (3) path displacement, (4) path deviation from straightness, defined as the area (Whole Deviation, WD) delimited by the virtual start-to-target straight line and the actual wrist path, and (5) path curvature, the length of the actual wrist path divided by the virtual start-to-target straight line.

The 11 markers also permitted us to compute the position of the whole body CoM using an eight-segment mathematical model consisting of the following rigid segments: head, trunk, thigh, shank, foot, upper arm, forearm, and hand. Using this model, the position of the CoM was calculated via standard procedures and using documented anthropometric parameters (Winter, 1990). An analogous model used to determine whole-body CoM position has previously been validated for similar whole-body pointing movements (Stapley, Pozzo, Cheron, \& Grishin, 1999). CoM displacements in the anteroposterior axis (A-P) were expressed as a percentage of the distance between markers placed at the 5 th metatarsophalangeal and the external malleolus of each participant's right foot. CoM displacements in vertical axis were expressed as a percentage of the distance between markers placed at the auditory meatus of the ear and the external malleolus of each participant's right foot.

We also computed the CoP displacements with respect to the same spatial reference system used for the kinematics data. Along the A-P axis, the CoP patterns were characterized by one peak in the backward direction, before the whole-body movement, and a second peak in the opposite direction, about at the completion of the reaching movement. We calculated the amplitude of the CoP displacement in the backward direction (onset backward-peak backward) as well as the amplitude of the whole $\mathrm{CoP}$ displacement (peak backward-peak forward). These displacements were expressed as a percentage of the distance between markers placed at the 5th metatarsophalangeal and the external malleolus of each participant's right foot.

Finally, we performed a PCA (Jolliffe, 1986) upon the time series of joint (angle between two adjacent segments-intrinsic reference) and elevation angular displacements (with regard to the vertical axis defined by gravity-extrinsic reference) to evaluate the whole-body movement coordination (see Figure 1). We used the correlation matrix to normalize our data set and to take into account the different range of motion of each segment. To account for different motor strategies separate PCA were performed for each participant and for each condition. Each PCA was performed on a correlation matrix of a data set composed of 48 columns (corresponding to eight angles across six trials) and 200 rows (normalized time, i.e., we consider that all movement durations are equal to 1 , and that all movements are composed by 200 frames). The eigenvectors of the correlation matrix represent a well adapted basis of the 48-dimensional linear space, characterizing the most important directions (in terms of percentage of variance accounted for) in the feature space. The first eigenvector (corresponding to the largest eigenvalue) represents the direction of the maximum variance in this space. The ratio between the first eigenvalue and all the eigenvalues (eight in total) gives a number between 0 and 1 (noted PC1\%). This value can be considered as an index of the movement coordination. A PC1\% of $100 \%$ will mean that all the angles together vary in a linear mode, that is, the trajectory in the space of angles is a straight line.

All measures showed normal distribution following a Shapiro-Wilk $\mathrm{W}$ test and equivalent variance (Levene test). Statistical effects were 
calculated by using ANOVA analysis with Group as a betweensubjects factor (Young, Elderly) and Speed within-participants factors (N, F). Post hoc differences were assessed by means of Scheffé tests. Reported results are considered significant for $p<.05$, not significant $p$ values are indicated in the text with (NS).

\section{Results}

\section{Main Experiment}

Figure 2A shows typical whole body reaching movements from one young and one elderly participant in both conditions of speed. In the intrinsic space, whole-body movements were characterized by large displacements $\left(>30^{\circ}\right)$ of knee, hip, back and shoulder joints and by smaller displacements of ankle, neck, elbow, and wrist joints in both conditions of speed (see Figure 2B). In the extrinsic space, elevation angles of thigh, pelvis, trunk, and head showed larger displacements than elevation angles of shank, humerus, forearm, and hand in both conditions of speed (see Figure 2C). ANOVA performed upon each joint and elevation angular displacements did not reveal main effect of Group or Speed and interaction effects between them (NS). Furthermore, PCA analysis (performed on joints and elevation angles) revealed a strong joint coupling for both groups and speed conditions. Individual values of PC1 ranged between $80 \%$ and $90 \%$. No main or interaction effects were found (NS) when comparing PC1 values among the different experimental conditions (for joint angles, Elderly Natural: $0.81 \pm 0.02$; Elderly Fast: $0.80 \pm 0.06$; Young Natural: $0.83 \pm$
0.05; Young Fast $0.85 \pm 0.05$ and elevation angles, Elderly Natural: $0.85 \pm 0.02$; Elderly Fast: $0.85 \pm 0.02$; Young Natural: $0.88 \pm 0.02$; Young Fast $0.85 \pm 0.04$ )

The comparison between the initial and the final body postures (see Figure 2A) revealed that all markers were oriented in the forward direction, except for the hip and the L5-S1 markers, which were oriented in the opposite direction (backward). However, these backward hip displacements were not sufficient to stabilize the CoM, which initially slightly moved forward and then forward and downward. Histograms in the Figure 3A depict average amplitudes of A-P CoM displacements. The statistical analysis gave a main effect of Speed $(F 1,14=30.44, p<.0001)$ and an interaction effect between Group and Speed $(F 1,14=21.04, p<$ .0001). The post hoc analysis showed a significant decrease in fast compared to normal velocity only for the young group $(p<.001)$ and a significant difference between young and elderly groups only for the natural movements $(p<.05)$. Histograms in the Figure 3B show average amplitudes of downward vertical (D-V) CoM displacements. The statistical analysis gave only a main effect of Group $(F 1,14=17.14, p<.01)$; CoM displacement along the vertical axis decreased in elderly, compared to young adults.

Figure 4A shows typical $\mathrm{CoP}$ displacements along the A-P axis from one young and one elderly participant. At normal speed, the first detectable mechanical event was a backward displacement of the CoP, followed by a large forward displacement, after which there were small adjustments of the final $\mathrm{CoP}$ position at the



Figure 2. (A) Typical whole body reaching movements from one young and one elderly participant. Wrist and center of mass $(\mathrm{CoM})$ displacements are also shown (gray lines). (B) Average displacements $( \pm S D)$ of joint angles. (C) Average displacements $( \pm S D)$ of elevation angles. 


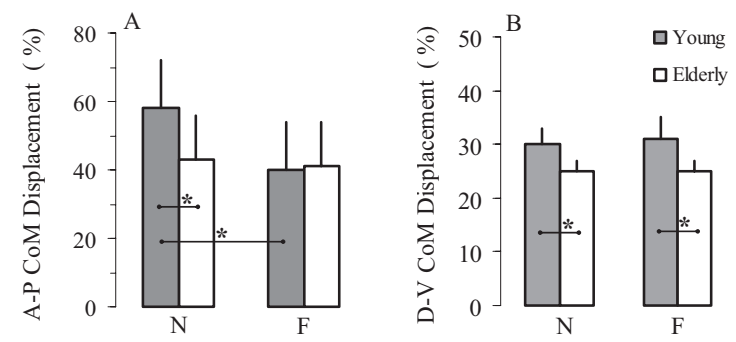

Figure 3. (A) Average amplitudes $( \pm S D)$ of CoM A-P displacements expressed as a percentage of the distance between markers placed at the 5th metatarsophalangeal and the external malleolus of each participant's left foot. (B) Average amplitudes $( \pm S D$ ) of CoM D-V displacements expressed as a percentage of the distance between markers placed at the auditory meatus of the ear and the external malleolus of each participant. Normal speed $(N)$; fast speed $(\mathrm{F})$.

forward end of the base of support (as previously recorded in (Stapley et al., 1999). Backward and forward displacements were greater for the young compared to the elderly participants. A second pattern could be identified during fast reaching movements, that is, two successive backward and forward displacements. Again, A-P CoP displacements were greater for the young than the elderly participants. Figure 4B shows average values of CoP backward displacements with respect to the initial position. The statistical analysis gave a main effect of Group $(F 1,14=16.15$, $p<.01)$ and Speed $(F 1,14=9.15, p<.01)$; interaction effects were not significant (NS). Figure $3 \mathrm{C}$ also illustrates average values of $\mathrm{CoP}$ displacements (amplitude between the backward and forward peaks). The statistical analysis gave only a main effect of Group $(F 1,14=5.57, p<.05)$.

Figure 2A depicts typical wrist paths in the sagittal plane. Generally, path curvature was different for the young compared to the elderly participants. Specifically, wrist paths of young participants were convex, whereas those of elderly participants were almost straight with the first part of the path to be slightly concave and the last slightly convex. In general, wrist path curvatures of young participants were larger compared to those of elderly participants. ANOVA, performed upon the total length of path displacements, gave a main effect of Group $(F 1,14=168.9, p<$ .0001). Speed main effect (NS) and interaction effects between the two factors were not significant (NS). Figure 5A illustrates average paths for the $(n=8)$ young and elderly $(n=8)$ groups and Figure $5 \mathrm{~B}$ shows the average values $( \pm S D)$ from the area of the wrist curvature. ANOVA, performed upon path area, gave only a main effect of Group $(F 1,14=12.28, p<.003)$; main effect of Speed (NS) and interaction effects were not significant (NS). We found similar results regarding path curvature. Precisely, ANOVA gave a main effect of Group $(F 1,14=37.02, p<.001)$ but not main effect of Speed (NS) or interaction effects (NS).

Figure 6 illustrates typical tangential velocity profiles for the two conditions of speed, which nicely illustrate the general temporal features of the wrist motion for the elderly and the young participants. Velocity profiles were bell-shaped and singled peaked. Movement durations were longer in the elderly compared to the young group; they increased by $20 \%$ and $30 \%$ at normal and at fast speed respectively. ANOVA gave a main effect of Group $(F 1,14=18.45, p<.001)$ and Speed $(F 1,14=67.74, p<.001)$; interaction effects were not significant (NS). Peak velocity was lower in the elderly compared to the young group; it decreased by $32 \%$ and $39 \%$ at normal and fast speed, respectively. ANOVA gave a main effect of $\operatorname{Group}(F 1,14=39.15, p<$ $.001)$ and Speed $(F 1,14=115.53, p<.001)$ interaction effects were not significant (NS).

\section{Control Experiment}

The main results of our experiment showed significant differences between young and elderly adults in parameters that characterized equilibrium (CoM and $\mathrm{CoP}$ ) and wrist trajectory (path and velocity) patterns. However, equilibrium and wrist trajectory are mechanically linked during the whole body reaching (WBR). Unstable equilibrium for instance, could cause a deviation of wrist path from the desired one and alternatively, large and fast wrist displacements could disturb equilibrium. Consequently, the causal
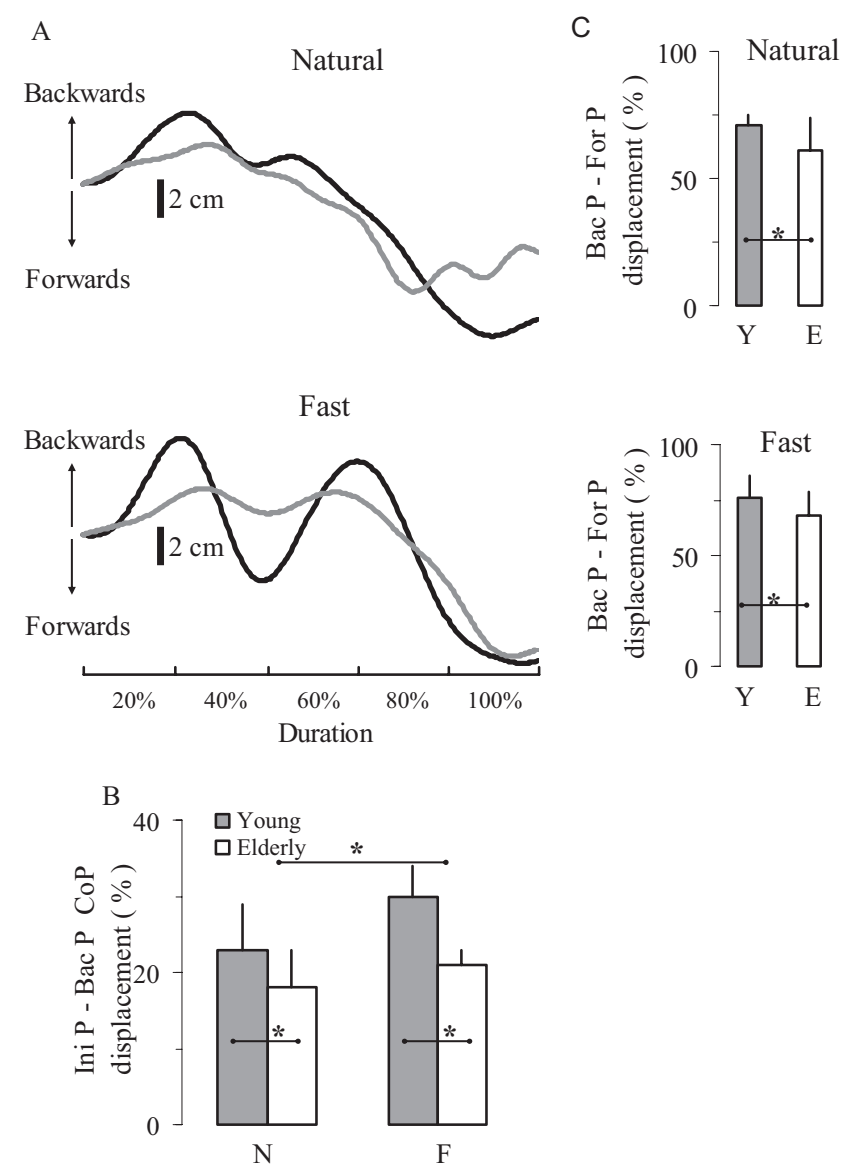

Figure 4. (A) Typical CoP displacements along the A-P axis for one young (black line) and one elderly (gray line) participant in both speed conditions. (B) Average amplitudes $( \pm S D)$ of $\mathrm{CoP}$ A-P displacements (measured between the initial position (Ini P) and the first backward peak [Bac P]) expressed as a percentage of the foot length (i.e., the distance between the markers placed at the 5 th metatarsophalangeal and the external malleolus). (C) Average amplitudes $( \pm S D)$ of the whole CoP A-P displacements (peak backward-peak forward [For P]) expressed as a percentage of the foot length. Young (Y); elderly (E). 

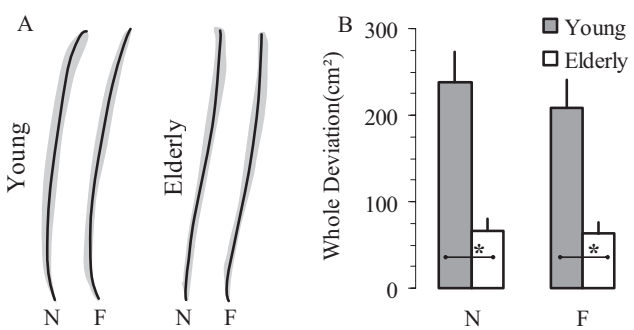

Figure 5. (A) Average and normalized (in amplitude and direction) wrist paths (black lines) for the Young and the Elderly group. Gray indicates standard deviation. (B) Average values $( \pm S D)$ of wrist path curvature, whole deviation (WD), defined as the area delimited by the virtual startto-target straight line and the actual wrist path.

relationship between equilibrium and wrist trajectory changes because of ageing is rather difficult to determine. To clarify this issue we designed control experiments where young adults were asked to reach the target under focal and postural constraints.

In agreement with previous studies (Ketcham et al., 2002; Narici et al., 2003; Personnier, Paizis, Ballay, \& Papaxanthis, 2008; P. Reuter-Lorenz, 2002; P. A. Reuter-Lorenz \& Lustig, 2005; Skoura, Papaxanthis, Vinter, \& Pozzo, 2005; Verschueren et al., 2002) we formulate the hypothesis that elderly adults adapt their motor repertoire to the age-related alterations of sensorimotor function (proprioception, muscular mechanics, cognitive processes). In particular, we postulate that the preservation of equilibrium is the primary goal of elderly adults during whole body movements and that this internal constraint instigates modifications in the control of postural ( $\mathrm{CoM}$ and $\mathrm{CoP}$ ) and focal (wrist trajectory formation) components of the task. To validate this hypothesis, we compared the behavior of the elderly adults with that of young adults who were asked to perform the WBR under equilibrium and spatial constraints. We anticipated that only experimentally induced equilibrium constraints in young adults will simulate an elderly strategy consisting in a modification of wrist kinematics and in a reduction of CoM displacement (see results of the main experiment). We considered that this hypothesis would be valid if and



Figure 6. Typical wrist velocity profiles for one young and one elderly participant in both speed conditions. only if: (1) under equilibrium constraints young adults showed wrist kinematics similar to those recorded in elderly adults (i.e., straight paths) and (2) under spatial constraints CoM patterns of young adults did not reproduce those recorded in elderly adults (i.e., reduced A-P displacements).

We carried out two control experiments with the same young adults who participated in the main experiment. The data were analyzed using similar methods as in the main experiment and were later compared with the data previously recorded in elderly adults (i.e., during the main experiment). The equilibrium constraint (EC condition) consisted of placing the young participants on a reduced base of support composed of a $40 \mathrm{~cm}$ large square (wooden board) fixed on a thin length of wood $(5 \mathrm{~cm}$ high, $5 \mathrm{~cm}$ large, and $40 \mathrm{~cm}$ length, see Figure 7A). Participants were requested to perform the same motor task without falling from the support. We paid attention to the stability of the supporting board during the recording session and trials $(n=4)$ where the board rotated forward or backward and touched the ground were rejected.



B



- - Young

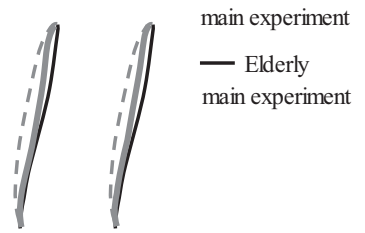

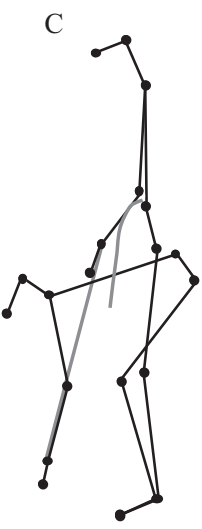

$\mathrm{D}$

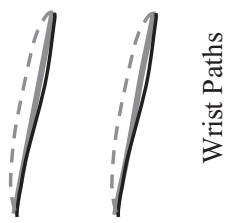

Figure 7. (A) Equilibrium constraints (EC). Stick diagrams indicating the initial and final body positions of one typical young adult performing a WBR movement at natural speed from a reduced base of support. (B) Average CoM and wrist path displacement in the sagittal plane from the main experiment (group of elderly and group of young) and the control experiment (EC) are superimposed. (C) Spatial constraints (SC). Stick diagrams indicating the initial and final body positions of one typical young adult performing a WBR movement at natural speed under spatial constraints (i.e., straight wrist path). (D) Average CoM and wrist path displacement in the sagittal plane from the main experiment (group of elderly and group of young) and the control experiment (SC) are superimposed. 
In the spatial constraint (SC condition), young participants were requested to reach the targets by using a straight finger trajectory. Participants initially performed three nonrecorded trials by tracking a straight wire connecting the initial finger position to the target. After this short period, they were asked to perform the task (without the presence of the wire) and were suggested to keep the same speed as in the main experiment.

Figure 7A shows a typical WBR movement, executed at a natural speed, from a young adult under equilibrium constraints. In this condition, young adults significantly decreased (paired $t$ tests, $p<.001$ for both speeds) the A-P displacement of their CoM, compared with the main experiment (see Table 1). They also slightly, but significantly (paired $t$ tests, $p<.05$ for both speeds), modulated the $\mathrm{D}-\mathrm{V}$ displacement of their CoM. This behavior is qualitatively similar to that of elderly adults in the main experiment (see Figure 7B). Quantitatively, the A-P displacement of CoM, is a little smaller compared with the elderly (paired $t$ tests, $p<.05$ for both speeds). For the D-V displacement of CoM, statistical analysis did not show significant modifications (between group $t$ tests, NS; see Table 1).

Interestingly, decrease in CoM displacement under equilibrium constraints was associated with changes in wrist kinematics. Notably, young adults significantly decreased the curvature of wrist paths (paired $t$ tests, $p<.001$ for both speeds), compared with the main experiment. In this condition, path's curvature and path's deviation from straightness of young adults was not statistically different from that of elderly adults recorded in the main experiment (between group $t$ tests, NS). Wrist duration and peak velocity did not show significant modifications (paired $t$ tests, NS for both speeds) under equilibrium constraints (see Table 1) compared with the main experiment. Despite these modifications of CoM and path curvature under equilibrium constraints, young participants did not change the general strategy of coordination consisting of joint coupling. The PCA analysis did not reveal significant differences (paired $t$ tests, NS for both speeds) between the main and the equilibrium constraint experiments.

Figure 7C shows a typical WBR movement, executed at a natural speed, from one young adult under spatial constraints. As expected for this condition, young adults significantly decreased path's curvature and path's deviation from straightness in comparison with the main experiment (paired $t$ tests, $p<.0001$ for both speeds). Furthermore, path's curvature and path's deviation from straightness of young adults was not statistically different (between group $t$ tests, NS) from that of elderly adults recorded in the main experiment (see Table 1). Interestingly, imposed straight wrist path did not induce CoM adaptations (see Figure 7D). Young adults did not change the A-P and D-V displacements of their CoM between the main and the spatial constraint experiments (paired $t$ tests, NS for both speeds). Wrist duration and peak velocity did not show significant modifications (paired $t$ tests, NS for both speeds) under wrist trajectory constraints (see Table 1). Changes in path curvature in young participants did not affect the movement coordination evaluated through joint co variation; the PCA analysis did not reveal significant differences (paired $t$ tests, NS for both speeds) between the main and the control experiment.

\section{Discussion}

The main finding of the current study is that during WB movements, CoM displacements are smaller in elderly compared to young adults and that this postural aging effect is associated with a concomitant focal effect, that is straighter wrist paths. Despite these changes, high covariations of joint and elevation angles, observed in young adults, were also preserved in old adults. In the following discussion we will highlight the specificities of dynamic equilibrium that requires a fine integration of postural and focal modules of the task in aging. We will also formulate hypotheses concerning the potential causes that explain the underlying mechanism of the modification of postural strategy in aging.

\section{Elderly Participants' CoM Displacements Are Smaller Compared to Those of Young Adults}

An important result of this study is that during a WBR task beyond arm length, elderly subjects, compared to young subjects, restrict their $\mathrm{CoM}$ and $\mathrm{CoP}$ displacements along the A-P axis. Restricting displacement of the CoM is probably the way to counteract potential falls when one has lost flexibility in postural reactions. Indeed, the analysis of APAs recorded in elderly participants performing an arm raising task have revealed a decreased movement efficiency, delayed anticipatory preparation for movement and impaired coordination of postural adjustments compared

Table 1

Average Values of Spatial and Temporal Parameters of WBR Movements

\begin{tabular}{|c|c|c|c|c|c|c|c|c|}
\hline & \multicolumn{4}{|c|}{ Main experiment } & \multicolumn{4}{|c|}{ Control experiment } \\
\hline & \multicolumn{2}{|c|}{ Free of constraints } & \multicolumn{2}{|c|}{ Free of constraints } & \multicolumn{2}{|c|}{ Equilibrium constraints } & \multicolumn{2}{|c|}{ Spatial constraints } \\
\hline & \multicolumn{2}{|c|}{ Elderly } & \multicolumn{2}{|c|}{ Young } & \multicolumn{2}{|c|}{ Young } & \multicolumn{2}{|c|}{ Young } \\
\hline & Natural & Fast & Natural & Fast & Natural & Fast & Natural & Fast \\
\hline Duration sec & $0.98 \pm 0.20$ & $0.66 \pm 0.06$ & $0.79 \pm 0.10$ & $0.45 \pm 0.04$ & $0.81 \pm 0.04$ & $0.46 \pm 0.05$ & $0.80 \pm 0.04$ & $0.46 \pm 0.04$ \\
\hline Velocity $\mathrm{m} / \mathrm{s}$ & $1.16 \pm 0.16$ & $1.69 \pm 0.39$ & $1.71 \pm 0.26$ & $2.81 \pm 0.30$ & $1.63 \pm 0.09$ & $2.71 \pm 0.23$ & $1.61 \pm 0.05$ & $2.69 \pm 0.17$ \\
\hline Wrist displacements $\mathrm{cm}$ & $70.7 \pm 5$ & $70 \pm 5$ & $78.6 \pm 6.7$ & $78.5 \pm 5.9$ & $72.8 \pm 3.4$ & $73.2 \pm 2.8$ & $69.1 \pm 1.9$ & $69.3 \pm 1.6$ \\
\hline Wrist paths WD $\left(\mathrm{cm}^{2}\right)$ & $66.5 \pm 13$ & $62 \pm 16$ & $238 \pm 30$ & $208.6 \pm 33$ & $90.6 \pm 6$ & $75 \pm 15$ & $44.1 \pm 2.1$ & $46.3 \pm 2.3$ \\
\hline Wrist curvature & $1.07 \pm 0.04$ & $1.07 \pm 0.03$ & $1.18 \pm 0.04$ & $1.18 \pm 0.02$ & $1.09 \pm 0.04$ & $1.10 \pm 0.02$ & $1.03 \pm 0.04$ & $1.04 \pm 0.02$ \\
\hline CoM A-P \% & $43 \pm 13$ & $41 \pm 13$ & $58 \pm 14$ & $40 \pm 14$ & $22 \pm 3$ & $18 \pm 3$ & $59 \pm 14$ & $41 \pm 15$ \\
\hline CoM D-V \% & $25 \pm 2$ & $25 \pm 2$ & $30 \pm 3$ & $31 \pm 4$ & $24 \pm 2$ & $25 \pm 3$ & $29 \pm 2$ & $30 \pm 2$ \\
\hline
\end{tabular}


with young controls (Inglin \& Woollacott, 1988; Stelmach, Phillips, DiFabio, \& Teasdale, 1989). On the other hand, one could have predicted that the alteration of the motor and sensory systems with advance in age could have generated a more variable, possibly greater CoP displacement (Corbeil, Blouin, Begin, Nougier, \& Teasdale, 2003; Nardone, Tarantola, Giordano, \& Schieppati, 1997). Our findings show that older individuals are aware of their postural imbalance and limitations in postural reactions and are able to compensate for them by decreasing the displacement of their CoM.

\section{Motor Strategy Adopted by Elderly Participants}

With normal aging emerges sarcopenia (Narici et al., 2003), reduction in motor unit activation capacity (Winegard, Hicks, Sale, \& Vandervoort, 1996), coactivation of antagonist muscles (Klein et al., 2001; Seidler et al., 2002), decline in several aspects of proprioceptive sensitivity (Cheron \& Bengoetxea, 2006; Palluel, Nougier, \& Isabelle, 2008; Verschueren et al., 2002) and reduction in the central mechanisms operating during visuomotor information processing or motor imagery (Briggs, Raz, \& Marks, 1999; Personnier et al., 2008; Skoura et al., 2005). We think that these age-related physiological and cognitive modifications within the musculo-skeletal apparatus and the CNS, contribute to the alternations of the whole body movement planning and control observed in our study in aged individuals. Precisely, we consider that equilibrium preservation during whole body movements becomes an important parameter with age and, as equilibrium and wrist trajectory are mechanically linked, this internal constraint modifies the control of postural (CoM and CoP) and focal (wrist trajectory formation) components of the task. In our study, we found noticeable changes in wrist paths curvature and CoM displacements in elderly participants compared to young participants. As has been previously demonstrated (Pozzo, Ouamer, \& Gentil, 2001; Pozzo, Stapley, \& Papaxanthis, 2002; Stapley, Pozzo, \& Grishin, 1998) arm elevation favors the forward displacement of the CoM that in turn facilitates the use of gravity to accelerate the body toward the target but increases at the same time the whole-body horizontal momentum (Stapley et al., 1999). Here, flattening wrist paths performed by elderly adults induced a smaller forward displacement of the CoM and consequently helped to minimize the forward whole-body momentum and ankle torque. We think that these modifications rather than indicating a decline in motor control, suggest an adaptation to the continuous physiological changes associated with ageing. The results of the control experiment are in agreement with our assumption about the development of motor adaptation in elderly participants. Young adults were asked to reach the target from a reduced base of support, imposing a limited A-P CoM displacement that mimicked that of elderly participants. In the reduced base of support (BoS) condition young adults produced straighter wrist trajectory similar to that recorded in elderly adults. In contrast, when a straight wrist trajectory was imposed, young adults did not change the CoM displacement. This result indicates that wrist trajectory formation is equilibrium dependent and that such a hierarchy is included in the motor plan adopted by elderly adults (that is an effect of equilibrium on focal module but not of spatial constraints on equilibrium module). In other words, because wrist trajectory depends on equilibrium context we speculate that CoM stabilization recorded in elderly par- ticipants corresponds to an adaptative strategy that is reproduced by young adults performing WBR under equilibrium constraints. Interestingly, muscular fatigue of the lower limbs during whole body pointing in young adults influences postural control (decrease of CoM displacement) and focal movement (straight finger path) in a similar way as do the imposed equilibrium constraint in our control experiment (Schmid, Schieppati, \& Pozzo, 2006). It seems, therefore, that when the motor system of young adults is experimentally constrained, motor output (notably the CoM displacement) resembles that of elderly subjects when they execute the same task without constraints. For all these reasons, we propose that the smaller CoM displacement recorded in elderly adults is because of a motor strategy chosen by elderly adults to adapt the motor command with respect to intrinsic physiological changes because of normal aging. This premise is supported by the fact that the PC analysis of joint and elevation angles revealed a single coordinative structure from foot to finger where distal and proximal body segments together contribute to simple movements in the space of angles. This result indicates that coordination between focal and postural components of the task is not altered by normal aging mechanisms and that kinematic synergy (i.e., the afore coordinative structure) is maintained in aging adults despite different CoM displacements and wrist trajectories. Single peaked and bell-shaped hand tangential velocity curves also corroborate this idea.

CoM stabilization may be an adaptative, but not an efficient, strategy. By reducing CoM displacement, elderly participants loose several advantages provided by forward acceleration. For instance, horizontal CoM displacements permitted the use of gravitational force (Stapley, Pozzo, Grishin, \& Papaxanthis, 2000). A forward CoM displacement generates an ankle gravitational torque (body mass multiplied by the distance between the CoM and the axis of ankle rotation) that, in turn, accelerates a forward fall. Indeed, assuming that the body has an inverted pendulum configuration, at least during the initiation phase, a stabilization of the CoM (at any position in the BoS) limits the creation of a moment arm between it and the axis of rotation, and thus prevents the generation and use of an ankle gravitational torque. By decreasing the A-P CoM displacement, elderly subjects decrease the whole body momentum and associated ankle muscle activity. A previous analysis of individual joint torques showed particularly that keeping the CoM at a constant horizontal position resulted in significantly smaller ankle peak flexor and extensor torques (Stapley et al., 2000). The weakness in muscular force (Narici et al., 2003; Simoneau et al., 2007) and the decline of proprioceptive sensitivity at the ankle joint (Verschueren et al., 2002), associated with the general slowness of movement preparation and execution in elderly (Whipple et al., 1987), may explain why elderly participants adopted a strategy consisting of stabilizing the CoM during WBR movements.

From a clinical point of view, we speculate that the strategy adopted by elderly participants would lead to a substantial depression in motor function and to the development of a learned "non use" of the ankle joint. This idea has been previously proposed for patients after stroke who present a marked contraction in the size of the cortical representation of the limb and who report that movement of that extremity is difficult (Taub, Uswatte, \& Elbert, 2002). A strategy that consists of keeping the CoM near its initial position excludes the possibility of controlling for disturbing body 
oscillations during reaching execution (Stapley et al., 2000). Not only does it prevents the use of the forward foot length as a dynamic equilibrium area, but also limits the control of any destabilization by the modulation of extensor muscular activity. As demonstrated for the standing posture (Gurfinkel, 1973) a forward CoM position during whole body reaching facilitates the use of soleus or gastrocnemius muscular activity to control for the disturbing effects of hand trajectory formation or forward trunk displacements. Based on the present observations, we think that an excessive control of CoM in elderly adults could produce progressive equilibrium deficiency, especially when the motor task requires whole body coordination.

\section{References}

Alexander, N. B. (1994). Postural control in older adults. Journal of the American Geriatrics Society, 42, 93-108.

Amiridis, I. G., Hatzitaki, V., \& Arabatzi, F. (2003). Age-induced modifications of static postural control in humans. Neuroscience Letters, 350, 137-140.

Belen'kii, V. E., Gurfinkel, V. S., \& Pal'tsev, E. I. (1967). [Control elements of voluntary movements]. Biofizika, 12, 135-141.

Bellgrove, M. A., Phillips, J. G., Bradshaw, J. L., \& Gallucci, R. M. (1998). Response (re-)programming in aging: A kinematic analysis. Journals of Gerontology. Series A, Biological Sciences and Medical Sciences, 53, M222-M227.

Briggs, S. D., Raz, N., \& Marks, W. (1999). Age-related deficits in generation and manipulation of mental images: I. The role of sensorimotor speed and working memory. Psychology and Aging, 14, 427-435.

Cheron, G., \& Bengoetxea, A. (2006). Vieillissement et controle cerebral de l'exercice. Science \& Sports, 21, 204.

Corbeil, P., Blouin, J. S., Begin, F., Nougier, V., \& Teasdale, N. (2003). Perturbation of the postural control system induced by muscular fatigue. Gait Posture, 18, 92-100.

Gurfinkel, V. S. (1973). Muscle afferentation and postural control in man. Agressologie, 14, 1-8.

Horak, F., \& McPherson, J. (1996). Postural orientation. In S. J. Rowel LB (Ed.), Handbook of physiology (Vol. 12, chap. 7, pp. 254-292): American Physiological Society.

Inglin, B., \& Woollacott, M. (1988). Age-related changes in anticipatory postural adjustments associated with arm movements. Journal of Gerontology, 43, M105-M113.

Jolliffe, I. T. (1986). Principal components analysis. New York: SpringerVerlag.

Ketcham, C. J., Seidler, R. D., Van Gemmert, A. W., \& Stelmach, G. E. (2002). Age-related kinematic differences as influenced by task difficulty, target size, and movement amplitude. Journals of Gerontology. Series B, Psychological Sciences and Social Sciences, 57, P54-P64.

Klein, C. S., Rice, C. L., \& Marsh, G. D. (2001). Normalized force, activation, and coactivation in the arm muscles of young and old men. Journal of Applied Physiology, 91, 1341-1349.

Lee, W. A. (1980). Anticipatory control of postural and task muscles during rapid arm flexion. Journal of Motor Behavior, 12, 185-196.

Maki, B. E. (1993). Biomechanical approach to quantifying anticipatory postural adjustments in the elderly. Medical and Biological Engineering and Computing, 31, 355-362.

Maki, B. E., Cheng, K. C., Mansfield, A., Scovil, C. Y., Perry, S. D., Peters, A. L., et al. (2008). Preventing falls in older adults: New interventions to promote more effective change-in-support balance reactions. Journal of Electromyography and Kinesiology, 18, 243-254.

Medell, J. L., \& Alexander, N. B. (2000). A clinical measure of maximal and rapid stepping in older women. Journals of Gerontology. Series A, Biological Sciences and Medical Sciences, 55, M429-M433.
Morasso, P. G., Baratto, L., Capra, R., \& Spada, G. (1999). Internal models in the control of posture. Neural Networks, 12, 1173-1180.

Mourey, F., Pozzo, T., Rouhier-Marcer, I., \& Didier, J. P. (1998). A kinematic comparison between elderly and young subjects standing up from and sitting down in a chair. Age and Ageing, 27, 137-146.

Nardone, A., Tarantola, J., Giordano, A., \& Schieppati, M. (1997). Fatigue effects on body balance. Electroencephalography and Clinical Neurophysiology, 105, 309-320.

Narici, M. V., Maganaris, C. N., Reeves, N. D., \& Capodaglio, P. (2003). Effect of aging on human muscle architecture. Journal of Applied Physiology, 95, 2229-2234.

Palluel, E., Nougier, V., \& Isabelle, O. (2008). Do spike insoles enhance postural stability and plantar-surface cutaneous sensitivity in the elderly? $A G E, 30,53-61$.

Personnier, P., Paizis, C., Ballay, Y., \& Papaxanthis, C. (2008). Mentally represented motor actions in normal aging II. The influence of the gravito-inertial context on the duration of overt and covert arm movements. Behavioural Brain Research, 186, 273-283.

Pozzo, T., Ouamer, M., \& Gentil, C. (2001). Simulating mechanical consequences of voluntary movement upon whole-body equilibrium: The arm-raising paradigm revisited. Biological Cybernetics, 85, $39-$ 49.

Pozzo, T., Stapley, P. J., \& Papaxanthis, C. (2002). Coordination between equilibrium and hand trajectories during whole body pointing movements. Experimental Brain Research, 144, 343-350.

Reuter-Lorenz, P. (2002). New visions of the aging mind and brain. Trends in Cognitive Science, 6, 394.

Reuter-Lorenz, P. A., \& Lustig, C. (2005). Brain aging: Reorganizing discoveries about the aging mind. Current Opinion in Neurobiology, 15, 245-251.

Salthouse, T. A., \& Somberg, B. L. (1982). Isolating the age deficit in speeded performance. Journal of Gerontology, 37, 59-63.

Schmid, M., Schieppati, M., \& Pozzo, T. (2006). Effect of fatigue on the precision of a whole-body pointing task. Neuroscience, 139, 909-920.

Seidler, R. D., Alberts, J. L., \& Stelmach, G. E. (2002). Changes in multi-joint performance with age. Motor Control, 6, 19-31.

Seidler-Dobrin, R. D., \& Stelmach, G. E. (1998). Persistence in visual feedback control by the elderly. Experimental Brain Research, 119, 467-474.

Simoneau, E. M., Billot, M., Martin, A., \& Van Hoecke, J. (2007). Antagonist mechanical contribution to resultant maximal torque at the ankle joint in young and older men. Journal of Electromyography and Kinesiology (Epub ahead of print).

Skoura, X., Papaxanthis, C., Vinter, A., \& Pozzo, T. (2005). Mentally represented motor actions in normal aging. I. Age effects on the temporal features of overt and covert execution of actions. Behavioural Brain Research, 165, 229-239.

Stapley, P., Pozzo, T., \& Grishin, A. (1998). The role of anticipatory postural adjustments during whole body forward reaching movements. Neuroreport, 9, 395-401.

Stapley, P., Pozzo, T., Grishin, A., \& Papaxanthis, C. (2000). Investigating centre of mass stabilisation as the goal of posture and movement coordination during human whole body reaching. Biological Cybernetics, 82 , 161-172.

Stapley, P. J., Pozzo, T., Cheron, G., \& Grishin, A. (1999). Does the coordination between posture and movement during human whole-body reaching ensure center of mass stabilization? Experimental Brain Research, 129, 134-146.

Stelmach, G. E., Phillips, J., DiFabio, R. P., \& Teasdale, N. (1989). Age, functional postural reflexes, and voluntary sway. Journal of Gerontology, 44, B100-B106.

Taub, E., Uswatte, G., \& Elbert, T. (2002). New treatments in neurorehabilitation founded on basic research. Nature Reviews. Neuroscience, 3 $228-236$. 
Vaillant, J., Vuillerme, N., Janvey, A., Louis, F., Braujou, R., Juvin, R., et al. (2008). Effect of manipulation of the feet and ankles on postural control in elderly adults. Brain Research Bulletin, 75, 18-22.

Verschueren, S. M., Brumagne, S., Swinnen, S. P., \& Cordo, P. J. (2002). The effect of aging on dynamic position sense at the ankle. Behavioural Brain Research, 136, 593-603.

Whipple, R. H., Wolfson, L. I., \& Amerman, P. M. (1987). The relationship of knee and ankle weakness to falls in nursing home residents: An isokinetic study. Journal of the American Geriatrics Society, 35, 13-20.

Winegard, K. J., Hicks, A. L., Sale, D. G., \& Vandervoort, A. A. (1996). A 12-year follow-up study of ankle muscle function in older adults.
Journals of Gerontology. Series A, Biological Sciences and Medical Sciences, 51, B202-B207.

Winter, D. A. (1990). Biomechanics and motor control of human movement. New York: Wiley.

Woollacott, M. H., \& Manchester, D. L. (1993). Anticipatory postural adjustments in older adults: Are changes in response characteristics due to changes in strategy? Journal of Gerontology, 48, M64-M70.

Received March 24, 2008

Revision received June 18, 2008

Accepted June 18, 2008

\section{ORDER FORM}

Start my 2009 subscription to Behavioral Neuroscience ISSN: 0735-7044

\section{\$148.00 APA MEMBER/AFFILIATE}

$\$ 286.00$ INDIVIDUAL NONMEMBER

$\$ 980.00$ INSTITUTION

In DC add 5.75\% / In MD add 6\% sales tax

TOTAL AMOUNT DUE

Subscription orders must be prepaid. Subscriptions are on a calendar year basis only. Allow 4-6 weeks for delivery of the first issue. Call for international subscription rates.

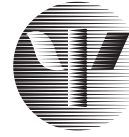

AMERICAN PSYCHOLOGICAL ASSOCIATION
SEND THIS ORDER FORM TO

American Psychological Association

Subscriptions

750 First Street, NE

Washington, DC 20002-4242

Call 800-374-2721 or 202-336-5600

Fax 202-336-5568 :TDD/TTY 202-336-6123

For subscription information,

e-mail: subscriptions@apa.org $\square$ Check enclosed (make payable to APA)

Charge my: $\square$ Visa $\square$ MasterCard $\square$ American Express

Cardholder Name

Card No Exp. Date

Signature (Required for Charge)

\section{Billing Address}

Street

City State Zip

Daytime Phone

E-mail

\section{Mail To}

Name

Address

City State Zip 